\title{
Modified Maag's Spherical Diffusion Model of Vacuum Penetration Grouting
}

\author{
Feng Huang (D), Jianguo Lyu (iD), He Gao, and Guihe Wang \\ School of Engineering \& Technology, China University of Geosciences, Beijing 100083, China \\ Correspondence should be addressed to Jianguo Lyu; ljg@cugb.edu.cn
}

Received 13 August 2017; Revised 11 December 2017; Accepted 25 December 2017; Published 21 January 2018

Academic Editor: Stefano de Miranda

Copyright (C) 2018 Feng Huang et al. This is an open access article distributed under the Creative Commons Attribution License, which permits unrestricted use, distribution, and reproduction in any medium, provided the original work is properly cited.

\begin{abstract}
The penetration grouting is very widely used in geotechnical engineering nowadays, but the slurry diffusion radius is not long enough because of low grouting pressure. The vacuum grouting method is proposed to solve this problem. However, there is no diffusion theory model of vacuum grouting, which makes the practical application lack scientific basis. In this paper, the distribution law of vacuum negative pressure in soil is deduced. Then, the boundary conditions of Maag's spherical diffusion model are modified by the vacuum pressure distribution law. After that, the vacuum modification model is deduced. Finally, Maag's model and modified model are analyzed according to a published experiment, which proves that the vacuum modification model is suitable for predicting the slurry diffusion of vacuum grouting. The proposed model provides a reference for the theoretical study of vacuum grouting.
\end{abstract}

\section{Introduction}

The sand layer which has the characteristics of loose structure, no cohesion, and poor self-stability is often encountered in geotechnical engineering. The sandy layer can cause severe damage to underground projects [1-3]. Grouting is a common method of soil reinforcement in underground engineering and has been playing an important role in treating the sand layer [4-7]. At present, penetration grouting method is widely used because of low grouting pressure and little disturbance to soil structure [8]. But at the same time, the low pressure leads to the short diffusion distance of slurry. However, if the grouting pressure rises excessively, it is easy to cause soil cracks, excessive deformation, and other consequences.

Therefore, researchers have been studying the mechanism of grouting pressure of slurry diffusion and analyzed the mechanical equilibrium mechanism of diffusion termination [9-11]. The researchers found that when the slurry was injected into the soil under constant grouting pressure, the slurry flow would slow down because of the decrease of pressure gradient and the increase of resistance, which results in the decline of shear stress. As soon as the shear stress declined to the yield stress of the slurry, the diffusion would be terminated [12]. Vacuum grouting method generates negative pressure field in soil. Under the condition of constant grouting pressure, the vacuum condition can increase the pressure gradient of the slurry, so as to increase the shear rate of slurry in diffusion process and make the shear stress larger than the yield stress, which will lead to the longer diffusion distance. Vacuum grouting can change the current situation where the traditional penetration grouting diffusion distance is insufficient.

Researchers in recent years have analyzed a lot about the grouting combined with vacuum in concrete structure field. Assaad and Daou [13] evaluated the effect of vacuum on the amount of water extracted along with resulting changes in grout properties including flowability, static yield stress, viscosity, unit weight, Wick-induced bleeding, and compressive strength. Assaad et al. [14] also presented that the partial or complete extraction of free mixing water due to vacuuming decreases flowability. In foundation treatment, Han et al. [15] presented a case study on the application of high pressure jet grouting pile to prevent vacuum preloading from leaking in the silty sand and silty clay layers. Peng et al. [16] proposed electroosmotic grouting coupled with vacuum drainage at the cathode. The experimental study shows that the treatment 
effect of electroosmotic grouting with vacuum drainage is not only better than general electroosmosis methods but also more uniform than such methods. Shen [17] obtained the relationship between the degree of vacuum and the size of the grouting body and found that the diffusion radii of the slurry grown with the increase of vacuum degree (absolute value of vacuum pressure) and grouting pressure. The present researches provide a useful way to explore the slurry diffusion mechanism of vacuum penetration grouting. However, the present studies only obtained the macro and qualitative law. They have not established the diffusion model of vacuum grouting and the relationship between the grouting parameters and the diffusion distance.

Therefore, this paper takes Newtonian fluid as the research object to deduce the diffusion model of vacuum penetration grouting on the basis of Maag's spherical diffusion model. The new diffusion model explains how the diffusion distance be enlarged in mathematical way and predicts the diffusion distance of the slurry by analysis formula by considering grouting coefficients. The vacuum modification model provides a reference to the scientific application of vacuum penetration grouting.

\section{Maag's Spherical Diffusion Model and Vacuum Modification Model}

Maag derived the diffusion formula of the grout in sand layer and proposed a spherical diffusion model of Newtonian fluid [17]. The theoretical assumptions are that the grout is Newton fluid when diffusing in sandy soil; the diffusion of slurry follows Darcy's law; the injected soil is homogeneous and isotropic; there is no dynamic water pressure when grouting in groundwater; the difference between the density of slurry and water is not considered; the viscosity of the slurry is constant during grouting and the slurry diffuses in spherical form starting from the end of injecting pipe.

Maag's spherical diffusion model is shown in Figure 1.

According to Darcy's law:

$$
Q=K_{g} i A t
$$

where $i=-d h / d r, K_{g}=K / \beta$, and $A=4 \pi r^{2}$.

According to the boundary conditions:

$$
\begin{aligned}
& r=r_{0}, \\
& h=H \\
& r=r_{1}, \\
& h=h_{0},
\end{aligned}
$$

Equation (3) can be obtained as follows by submitting the boundary conditions into Darcy's law:

$$
H-h_{0}=\frac{Q \beta}{4 \pi K t}\left(\frac{1}{r_{0}}-\frac{1}{r_{1}}\right) ;
$$

then $Q$ can be solved as:

$$
Q=\frac{4 \pi K t\left(H-h_{0}\right)}{\beta\left(1 / r_{0}-1 / r_{1}\right)} .
$$

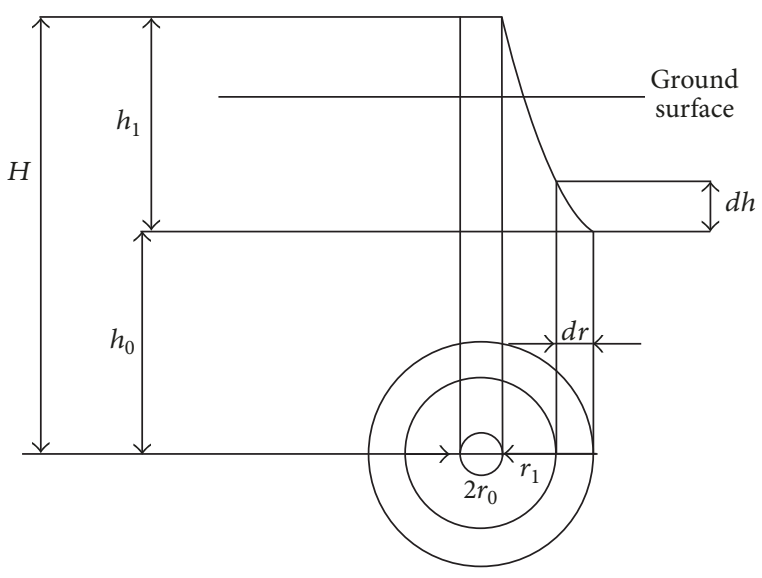

FIGURE 1: Schematic diagram of derivation of Maag's model.

Considering $r_{1}-r_{0} \approx r_{1}, 1 / r_{0}-1 / r_{1} \approx 1 / r_{0}$, and $H-h_{0}=$ $h_{1}$, as well as $Q=(4 / 3) \pi r_{1}^{3} n$, the expression of the diffusion radius of grouting can be derived as

$$
r_{1}=\sqrt[3]{\frac{3 K t r_{0} h_{1}}{n \beta}} .
$$

Q-total amount of grouting $\left(\mathrm{cm}^{3}\right)$;

$A$-slurry penetration area $\left(\mathrm{cm}^{2}\right)$;

$t$-total time for grouting (s);

$r$-the diffusion radius of the slurry at any moment (cm);

$r_{1}$-the final diffusion radius of the slurry $(\mathrm{cm})$;

$r_{0}$-radius of grouting pipe $(\mathrm{cm})$;

$h_{1}$-grouting pressure head $(\mathrm{cm})$;

$h_{0}$-water head of groundwater in the end of grouting pipe $(\mathrm{cm})$;

$H$-the sum of groundwater pressure head and grouting pressure head $(\mathrm{cm})$;

$n$-porosity of soil;

$\beta$ - the ratio of the viscosity of slurry to the viscosity of water, $\mu_{g} / \mu_{w}$;

$K$-permeability coefficient of injected soil $(\mathrm{cm} / \mathrm{s})$;

$K_{g}$-permeability coefficient for slurry $(\mathrm{cm} / \mathrm{s})$.

In order to study the influence of vacuum on the diffusion radius, the distribution of vacuum pressure in soil is analyzed firstly. The plane seepage model is used to derive the distribution formula of vacuum pressure. It is assumed that the seepage of air in soil is steady and follows Darcy's law. The vacuum pressure is converted into the corresponding water head through the derivation of the model, which can be coupled with the grouting pressure head. Finally, the new boundary conditions are submitted into Maag's spherical diffusion model and the expression of the diffusion radius of the slurry in vacuum condition is derived. 


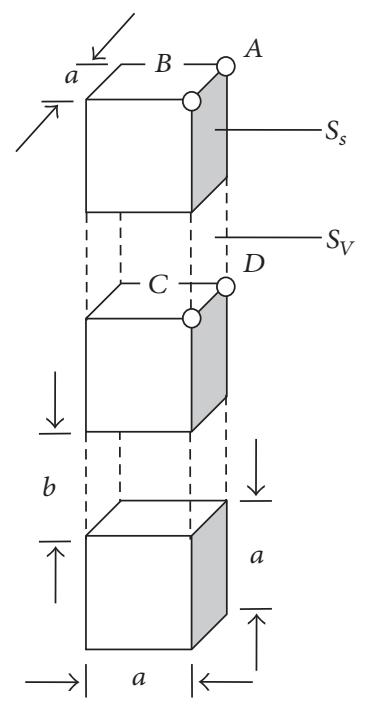

FIGURE 2: Soil particle and pore model.

Figure 2 shows the simplified model of soil established by particles and pores.

The soil porosity ratio $n$ is

$$
n=\frac{V_{a}}{V}=\frac{a^{2} b}{a^{2}(a+b)}=\frac{b}{(a+b)}
$$

while the porosity ratio $n_{s}$ of the soil section is

$$
n_{s}=\frac{S_{V}}{S_{V}+S_{s}}=\frac{a b}{a(a+b)}=\frac{b}{(a+b)} .
$$

It can be found that $n=n_{s}$ according to (6) and (7); the soil porosity can be represented by the porosity in the model. Although the change of effective stress and structure of soil caused by vacuum exists, the change is little for the real grouting projects especially in sand soil. Therefore, it is assumed that the soil structure is rigid.

The velocity of the air passing through a certain cross section of soil can be expressed as

$$
v=\frac{1}{r} \frac{q B_{g}}{2 \pi h n} .
$$

According to Darcy's law:

$$
\frac{d p}{d r}=\frac{\mu_{g}}{k_{g}} v=\frac{\mu_{g}}{k_{g}} \frac{1}{r} \frac{q B_{g}}{2 \pi h n} .
$$

Suppose $\left(\mu_{g} / k_{g}\right)\left(q B_{g} / 2 \pi h n\right)=C$. The control equation of the steady-state seepage in homogeneous porous media is

$$
d p=C \frac{1}{r} d r
$$

Then the following result is obtained:

$$
p=C C_{1} \ln r+C_{2} .
$$

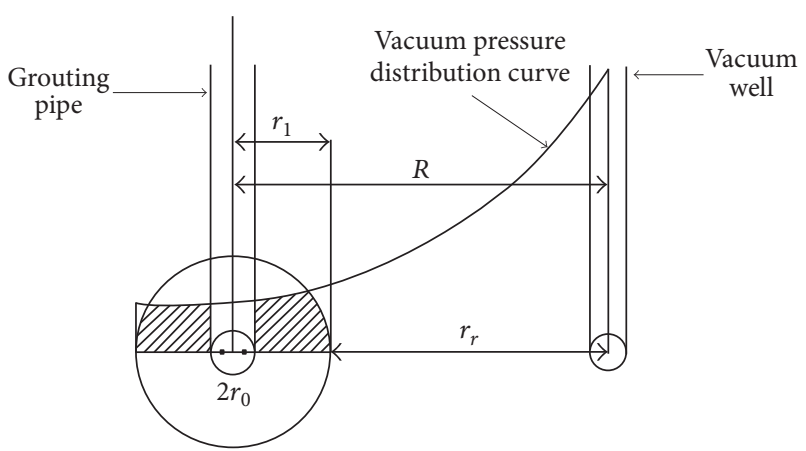

FIGURE 3: Schematic diagram of vacuum pressure distribution.

The boundary conditions are assumed as

$$
\begin{aligned}
& r=r_{r}, \\
& p=p_{r} \\
& r=r_{w}, \\
& p=p_{w} .
\end{aligned}
$$

In the conditions, the pressure $p_{r}$ is measured at a distance of $r_{r}$ from the vacuum well and $p_{w}$ of the vacuum well with a radius of $r_{w}$ is also measured. Then (11) can be expressed as

$$
\begin{aligned}
p_{w} & =C C_{1} \ln r_{w}+C_{2} \\
p_{r} & =C C_{1} \ln r_{r}+C_{2} .
\end{aligned}
$$

Finally, the distribution of the pressure $p$ along the distance $r$ caused by the vacuum well is

$$
p=\frac{p_{w}-p_{r}}{\ln \left(r_{w} / r_{r}\right)} \ln \left(\frac{r}{r_{w}}\right)+p_{w} .
$$

It is known that the $101 \mathrm{kPa}$ can be converted into the water head of about $10.3 \mathrm{~m}$, so the pressure difference $\Delta h$ caused by vacuum can be also expressed by water head based on the relationship between atmospheric pressure and water head. The water head caused by vacuum pressure is

$$
\Delta h=\frac{p_{0}-p_{w}}{9.806}-\frac{\left(p_{w}-p_{r}\right) \ln \left(r / r_{w}\right)}{9.806 \ln \left(r_{w} / r_{r}\right)} .
$$

The vacuum distribution is shown in Figure 3.

Because the seepage radius of slurry is comparatively much smaller than the range of vacuum pressure distribution, it is assumed that the vacuum pressure on the left and right side of the distance of $r_{1}$ is the same shown in Figure 3.

Assume

$$
\frac{p_{0}-p_{w}}{9.806}=C_{1}
$$

$$
\frac{p_{w}-p_{r}}{9.806 \ln \left(r_{w} / r_{r}\right)}=C_{2}
$$


Then Maag's spherical diffusion radius can be modified based on the boundary conditions as

$$
\begin{aligned}
r & =r_{0}, \\
h & =H-\Delta h_{0}, \\
\Delta h_{0} & =C_{1}-C_{2} \ln \left(\frac{R}{r_{w}}\right) \\
r & =r_{1}, \\
h & =H-\Delta h_{1}, \\
\Delta h_{1} & =C_{1}-C_{2} \ln \left(\frac{R-r_{1}}{r_{w}}\right) .
\end{aligned}
$$

The new boundary conditions are submitted into Darcy's law and the equations are integrated to obtain the diffusion radius under the vacuum effect:

$$
r_{1}^{\prime}=\sqrt[3]{\frac{3 K t r_{0}\left(h_{1}+\Delta h^{\prime}\right)}{n \beta}},
$$

where

$$
\Delta h^{\prime}=C_{2} \ln \left(\frac{R}{R-r_{1}}\right) \geq 0 .
$$

It can be seen from (18) that the vacuum pressure can enlarge the slurry diffusion distance because the vacuum pressure makes the water head boundaries lower than Maag's diffusion model.

\section{Verification Experiment of Vacuum Penetration Grouting}

According to the analysis above, the final diffusion radius of vacuum modification model will be influenced by many factors, like vacuum degree, soil conditions, and so on. In this paper, the vacuum modification formula is verified by the published vacuum grouting experiment by Shen [17].

3.1. Experimental Device and Materials. The vacuum grouting experiment system is presented in Figure 4.

The medium sand, fine sand, and silty sand were used as the injected stratum. The physical properties of experimental sand soil are shown in Table 1.

Acid sodium silicate solution was used in the experiment. The sodium silicate solution is $30^{\circ} \mathrm{Be}$ and the concentration of dilute sulphuric acid solution is $10 \%$. The volume ratio of sodium silicate solution to sulphuric acid is 0.7 . The gel time of the slurry is about $350 \mathrm{~s}$. The slurry is Newtonian fluid and the initial viscosity is $5 \mathrm{mPa} \cdot \mathrm{s}$.

3.2. Results and Discussion. In the experiment, $R$ is $0.2 \mathrm{~m}, r_{w}$ is $5 \mathrm{~cm}$, and $r_{0}$ is $1 \mathrm{~cm}$. The vacuum degree is $60 \mathrm{kPa}, 40 \mathrm{kPa}$, and $20 \mathrm{kPa}$ near the grouting pipe. The grouting lasted for $4.5 \mathrm{~min}$. The radii of the vacuum modification model are shown in Table 2.

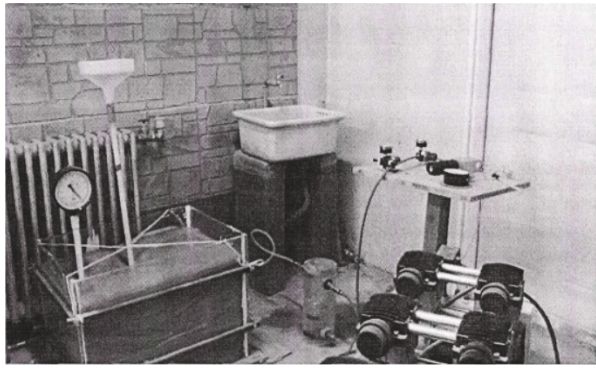

FIGURE 4: The experimental system [17].

TABle 1: Physical properties of sand samples.

\begin{tabular}{lccccc}
\hline Sand soil & $\rho\left(\mathrm{g} / \mathrm{cm}^{3}\right)$ & $K(\mathrm{~cm} / \mathrm{s})$ & $n(\%)$ & $\omega(\%)$ & $G\left(\mathrm{~g} / \mathrm{cm}^{3}\right)$ \\
\hline Medium & 1.61 & $1.45 \times 10^{-2}$ & 37.8 & 10.7 & 2.59 \\
Fine & 1.71 & $5.59 \times 10^{-3}$ & 34.5 & 12.5 & 2.61 \\
Silty & 1.79 & $9.75 \times 10^{-4}$ & 31.0 & 15.3 & 2.60 \\
\hline
\end{tabular}

Where $\rho$ is natural density; $K$ is permeability coefficient; $n$ is porosity; $\omega$ is moisture content; $G$ is specific gravity.

TABLE 2: Diffusion radii of Maag's spherical model and vacuum modification model.

\begin{tabular}{lccccc}
\hline$P_{r}(\mathrm{kPa})$ & Sand soil & $\Delta h^{\prime}(\mathrm{cm})$ & $r_{1}^{\prime}(\mathrm{mm})$ & $r_{1}(\mathrm{~mm})$ & $\Delta r(\mathrm{~mm})$ \\
\hline \multirow{4}{*}{60} & Medium & 251.5 & 136 & 85.9 & 50.1 \\
& Fine & 134.0 & 91 & 64.4 & 26.6 \\
& Silty & 60.6 & 48 & 36 & 12 \\
\hline \multirow{4}{*}{40} & Medium & 138.5 & 122 & 85.9 & 36.1 \\
& Fine & 82.7 & 86 & 64.4 & 21.6 \\
& Silty & 38.5 & 46 & 36 & 10 \\
\hline \multirow{3}{*}{20} & Medium & 58.7 & 110 & 85.9 & 24.1 \\
& Finer & 37.0 & 79 & 64.4 & 14.6 \\
& Silty & 18.3 & 44 & 36 & 8 \\
\hline
\end{tabular}

Where, $p_{r}$ is vacuum degree; $\Delta h^{\prime}$ is head change caused by vacuum; $r_{1}^{\prime}$ is radius of vacuum modification model; $r_{1}$ is radius of Maag's spherical model; $\Delta r$ is absolute difference between $r_{1}^{\prime}$ and $r_{1}$.

The comparison of diffusion radii in Maag's and vacuum modification models in vacuum condition is shown in Figures 5-7.

It is revealed from Figures 5-7 that, under the effect of vacuum negative pressure, the radii of Maag's spherical diffusion model are smaller than those of vacuum modification model. It illustrates that the vacuum grouting can enhance the diffusion ability of slurry by enlarging the diffusion radius. However, Maag's spherical diffusion model does not consider the effect of vacuum pressure and it can not be used to predict the slurry diffusion distance in vacuum condition.

The radii differences under different vacuum degrees are shown in Figure 8.

Figure 8 shows that the radii differences of vacuum modification model and Maag's model grow with the increases of the vacuum degree. It also can be seen that the larger the permeability coefficient of the soil, the larger the value of radius difference. 


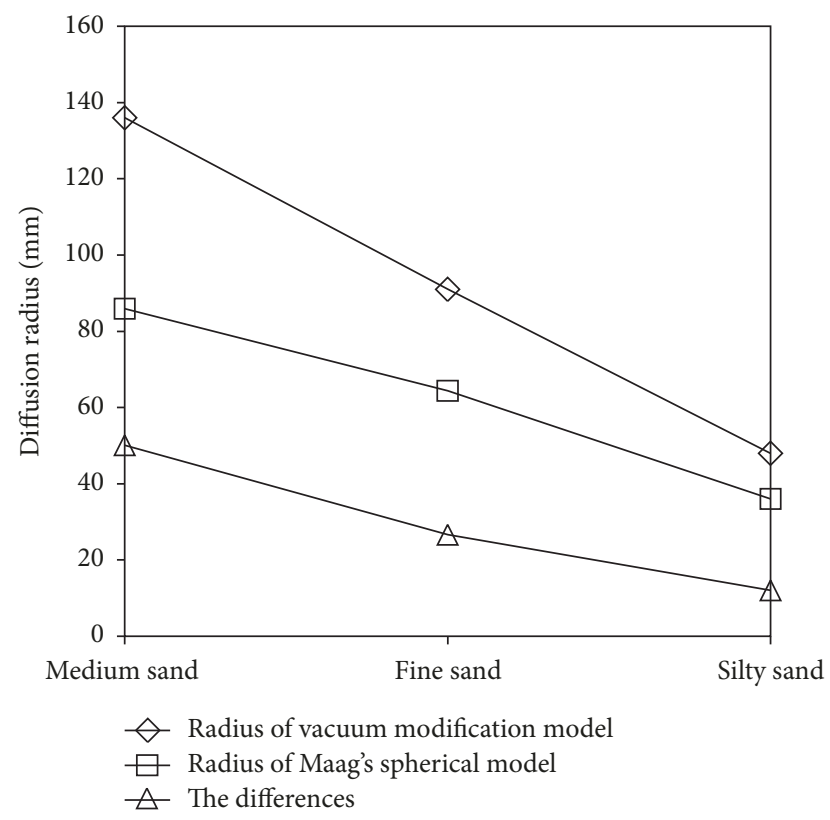

Figure 5: Diffusion radii under vacuum degree of $60 \mathrm{kPa}$.

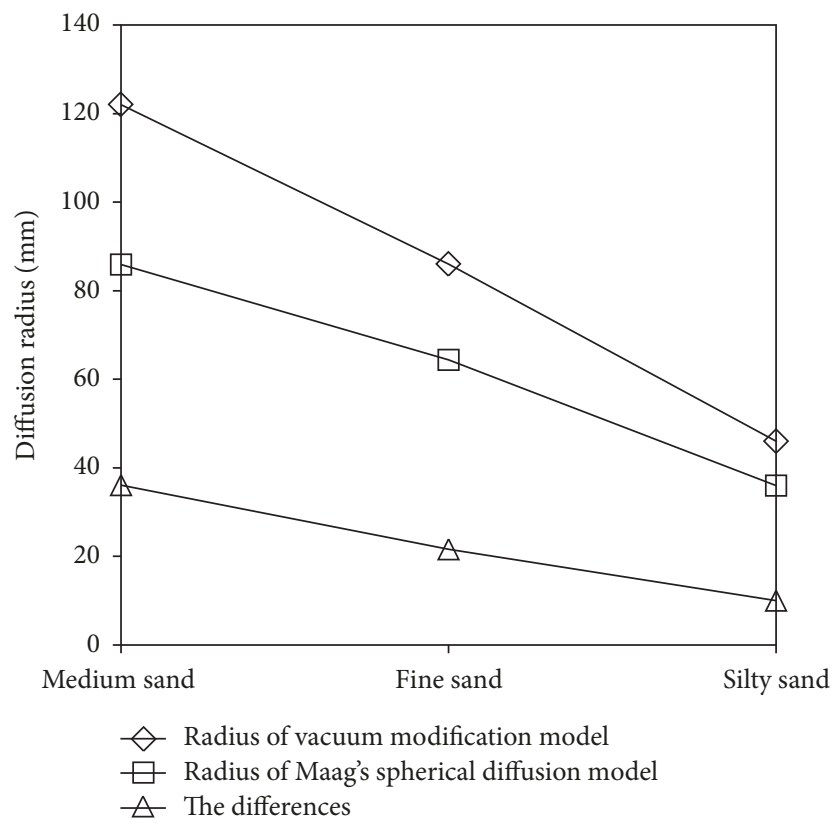

Figure 6: Diffusion radii under vacuum degree of $40 \mathrm{kPa}$.

In Shen's [17] experiment, the slurry was injected into 3 kinds of sand under vacuum degrees of $20 \mathrm{kPa}, 40 \mathrm{kPa}$, and $60 \mathrm{kPa}$, respectively. The theoretical values obtained by vacuum modification model and the corresponding measurement values in experiments are presented in Table 3.

The variation of experimental values and theoretical values is shown in Figures 9-11.

It can be obtained from Figures 9-11 that the trend of variation of the experimental diffusion radii is the same as that of the theoretical results. The diffusion radii of the grout

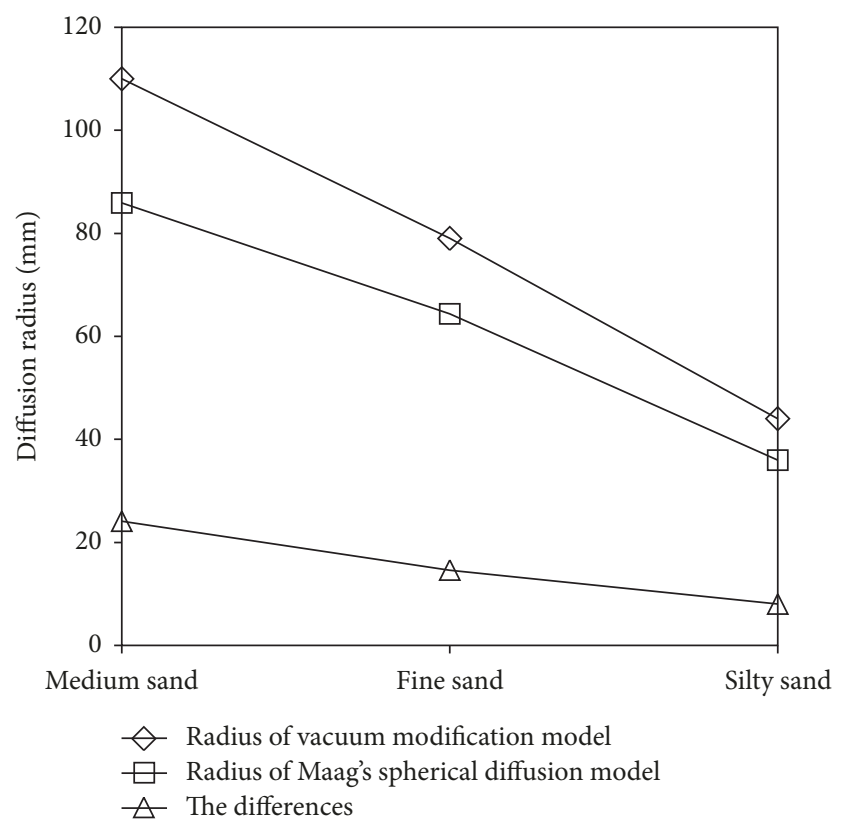

Figure 7: Diffusion radii under vacuum degree of $20 \mathrm{kPa}$.

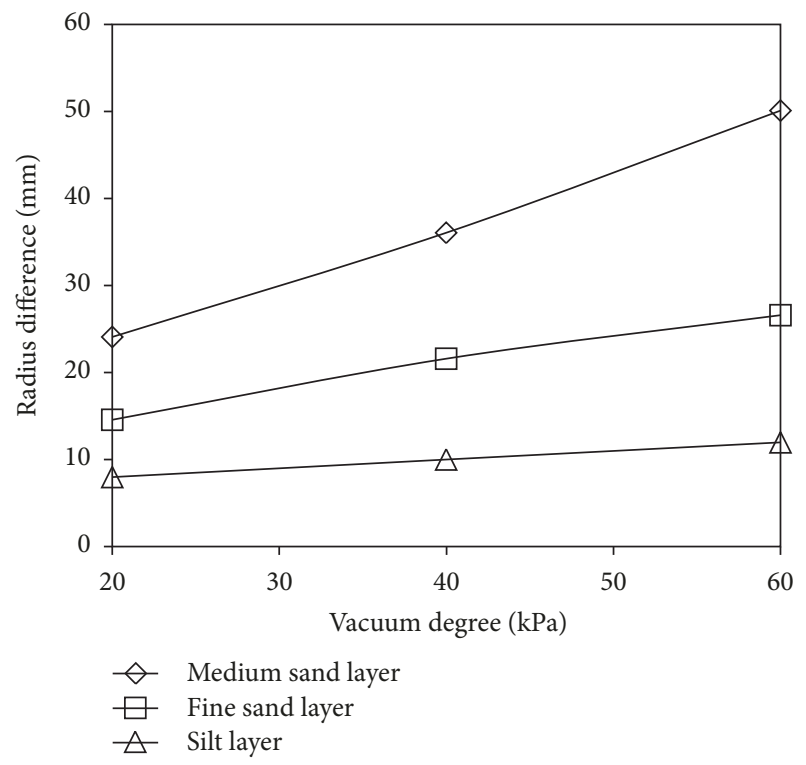

FIGURE 8: Radii differences under different vacuum degrees.

grow with the increase of the vacuum degree. The growth rate increases with the increase of permeability coefficient. The larger the vacuum degree, the larger the difference of the slurry diffusion radius. The curves of modification model are closer to the experiment values than the curves of Maag's model, especially in the silty layer. It also can be seen that the greater the vacuum degree, the smaller the deviations between the experimental values and the values of vacuum modification model. At the same time, the deviations between the experimental values and the values 
TABLE 3: Theoretical and experimental values of the diffusion radius under different vacuum degrees and soil conditions.

\begin{tabular}{lcccc}
\hline Sand stratum & $p_{r}(\mathrm{kPa})$ & $r_{1}^{\prime \prime}(\mathrm{mm})$ & $r^{\prime}(\mathrm{mm})$ & $\Delta r^{\prime}(\mathrm{mm})$ \\
\hline \multirow{3}{*}{ Medium } & 60 & 145 & 136 & -6.2 \\
& 40 & 90 & 122 & 35.5 \\
& 20 & 70 & 110 & 57.1 \\
\hline \multirow{3}{*}{ Fine } & 60 & 110 & 91 & -17.2 \\
& 40 & 80 & 86 & 7.5 \\
& 20 & 55 & 79 & 43.6 \\
\multirow{3}{*}{ Silty } & 60 & 75 & 48 & -36 \\
& 40 & 60 & 46 & -23.3 \\
& 20 & 45 & 44 & -2.2 \\
\hline
\end{tabular}

Where, $p_{r}$ is vacuum degree; $r_{1}^{\prime \prime}$ is experimental value; $r^{\prime}$ is theoretical value of modification model; $\Delta r^{\prime}$ is difference of $r_{1}^{\prime \prime}$ and $r_{1}$.

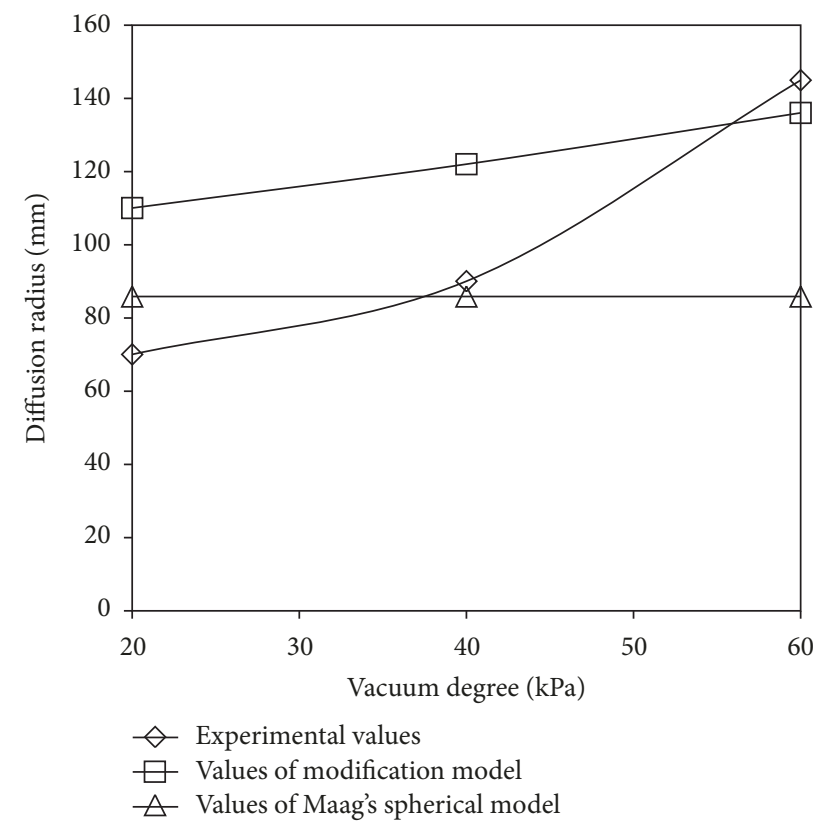

FIGURE 9: The variation of theoretical and experimental values in medium sand layer.

of Maag's model grow with the increases of vacuum degree. The differences between the vacuum modification values and the experimental values fall in the range of $-36 \%$ to $57 \%$. The theoretical model assumes that the soil is isotropic and the soil structure remains unchanged, but the soil used in experiment can not reach the ideal conditions. Due to the size limitation of the model box, the boundary conditions of experimental and theoretical models can not be completely consistent. Some studies show that the deviations between the actual measured values and the theoretical values are acceptable [18]. Therefore, the vacuum modification model is reasonable to predict the diffusion radius of vacuum penetration grouting.

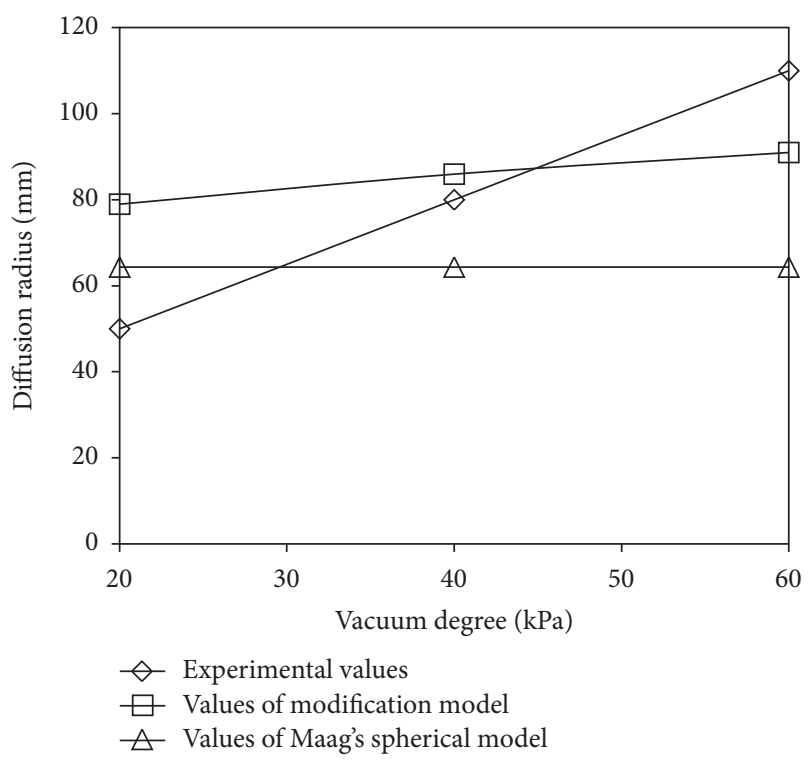

FIGURE 10: The variation of theoretical and experimental values in fine sand layer.

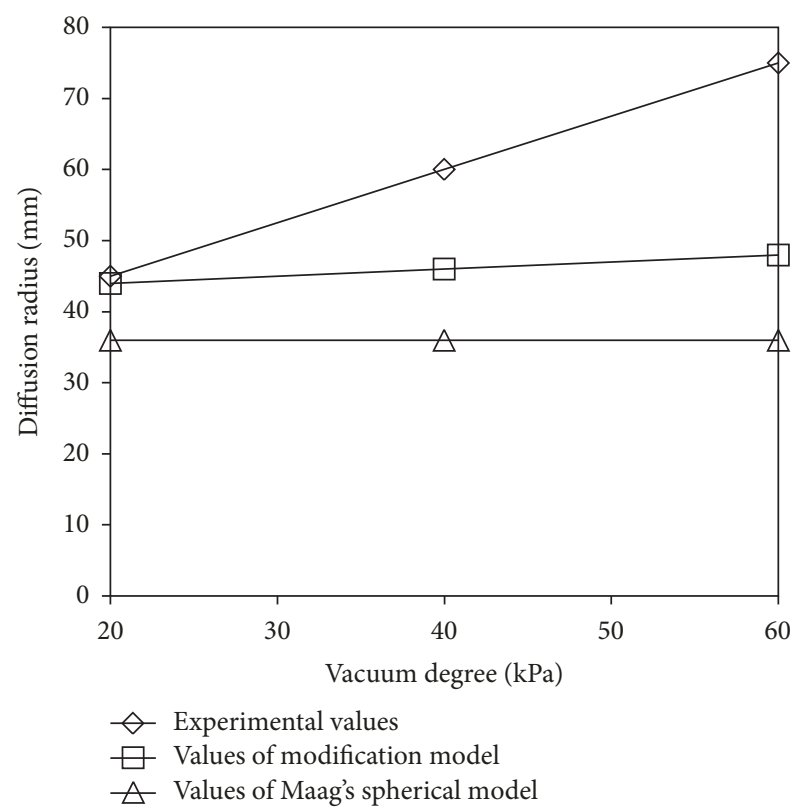

FIGURE 11: The variation of theoretical and experimental values in silt layer.

\section{Conclusions}

(1) Vacuum grouting method can enlarge the grout diffusion distance compared to the traditional method without vacuum pressure.

(2) It can be concluded from the theoretical analysis and experiment that the modified Maag's spherical diffusion model is more reasonable to predict the diffusion of grout than the traditional Maag's model. At the same time, it is shown in the vacuum modification model that the higher the 
absolute value of vacuum pressure, the larger the diffusion radius.

\section{Conflicts of Interest}

The authors declare that they have no conflicts of interest.

\section{Acknowledgments}

This study is supported by "The Fundamental Research Funds for the Central Universities of China” (no. 2-9-2015-082).

\section{References}

[1] G. Zhang, L. Song, and L. Chen, "A case study on treatment measures for a rock tunnel with inrush mud and sand gushing disaster," Disaster Advances, vol. 5, no. 4, pp. 489-493, 2012.

[2] C. A. Anagnostopoulos, "Laboratory study of an injected granular soil with polymer grouts," Tunnelling and Underground Space Technology, vol. 20, no. 6, pp. 525-533, 2005.

[3] G. Zheng, T. Cui, X. Cheng et al., "Study of the collapse mechanism of shield tunnels due to the failure of segments in sandy ground," Engineering Failure Analysis, vol. 79, pp. 464490, 2017.

[4] R. W. I. Brachman, C. D. Martin, and S. A. Gilliss, "Grout field trials in outwash sands," Canadian Geotechnical Journal, vol. 41, no. 1, pp. 1-11, 2004.

[5] W. Sui, J. Liu, W. Hu, J. Qi, and K. Zhan, "Experimental investigation on sealing efficiency of chemical grouting in rock fracture with flowing water," Tunnelling and Underground Space Technology, vol. 50, pp. 239-249, 2015.

[6] D. Neupane, H. Yasuhara, N. Kinoshita, and H. Putra, "Distribution of grout material within 1-m sand column in insitu calcite precipitation technique," Soils and Foundations, vol. 55, no. 6, pp. 1512-1518, 2015.

[7] Y. S. Kim and A. J. Whittle, "Particle network model for simulating the filtration of a microfine cement grout in sand," Journal of Geotechnical and Geoenvironmental Engineering, vol. 135, no. 2, pp. 224-236, 2009.

[8] J. Yoon and C. El Mohtar, "Groutability of granular soils using sodium pyrophosphate modified bentonite suspensions," Tunnelling and Underground Space Technology, vol. 37, pp. 135145, 2013.

[9] C. S. El Mohtar, J. Yoon, and M. El-Khattab, "Experimental study on penetration of bentonite grout through granular soils," Canadian Geotechnical Journal, vol. 52, no. 11, pp. 1850-1860, 2015.

[10] F. Bouchelaghem, L. Vulliet, D. Leroy, L. Laloui, and F. Descoeudres, "Real-scale miscible grout injection experiment and performance of advection-dispersion-filtration model," International Journal for Numerical and Analytical Methods in Geomechanics, vol. 25, no. 12, pp. 1149-1173, 2001.

[11] Z. Saada, J. Canou, L. Dormieux, J. C. Dupla, and S. Maghous, "Modelling of cement suspension flow in granular porous media," International Journal for Numerical and Analytical Methods in Geomechanics, vol. 29, no. 7, pp. 691-711, 2005.

[12] J. Yoon and C. S. El Mohtar, "A filtration model for evaluating maximum penetration distance of bentonite grout through granular soils," Computers \& Geosciences, vol. 65, pp. 291-301, 2015.
[13] J. J. Assaad and Y. Daou, "Cementitious grouts with adapted rheological properties for injection by vacuum techniques," Cement and Concrete Research, vol. 59, pp. 43-54, 2014.

[14] J. J. Assaad, Y. Daou, and J. Harb, "Influence of thixotropy on performance of grouts placed using vacuum injection techniques," ACI Materials Journal, vol. 112, no. 2, pp. 189-198, 2015.

[15] W.-J. Han, S.-Y. Liu, D.-W. Zhang, and G. Du, "Field behavior of jet grouting pile under vacuum preloading of soft soils with deep sand layer," in Proceedings of the GeoCongress 2012: State of the Art and Practice in Geotechnical Engineering, pp. 70-77, Geotechnical Special Publication, USA, March 2012.

[16] J. Peng, H. Ye, and A. N. Alshawabkeh, "Soil improvement by electroosmotic grouting of saline solutions with vacuum drainage at the cathode," Applied Clay Science, vol. 114, pp. 5360, 2015.

[17] G. L. Shen, Research on simulation test of vacuum grouting under different stratum, Chinese)., China University of Geosciences, Beijing, China, 2014.

[18] W.-J. Ruan, "Spreading model of grouting in rock mass fissures based on time-dependent behavior of viscosity of cement-based grouts," Chinese Journal of Rock Mechanics and Engineering, vol. 24, no. 15, pp. 2709-2714, 2005. 


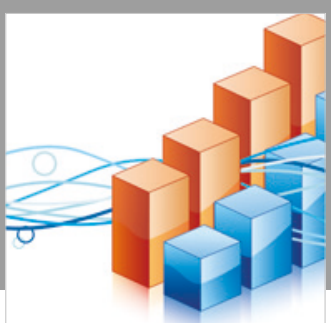

Advances in

Operations Research

\section{-n-m}
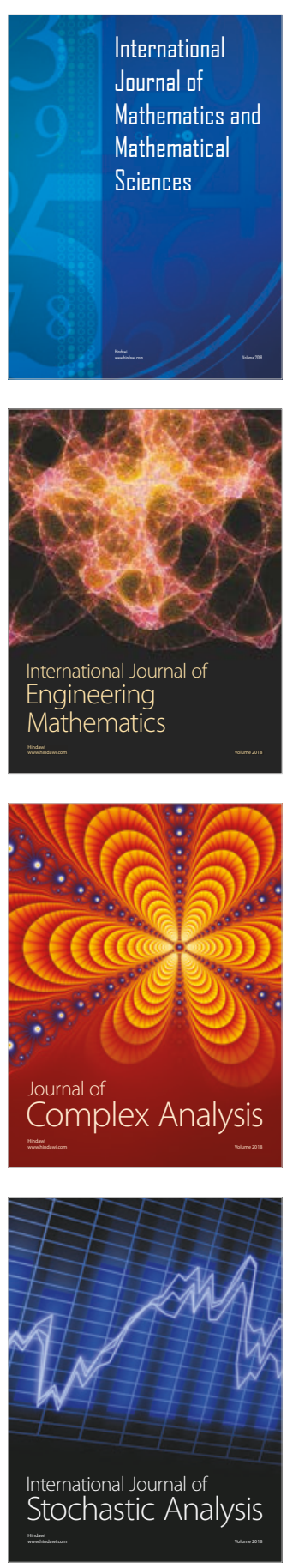
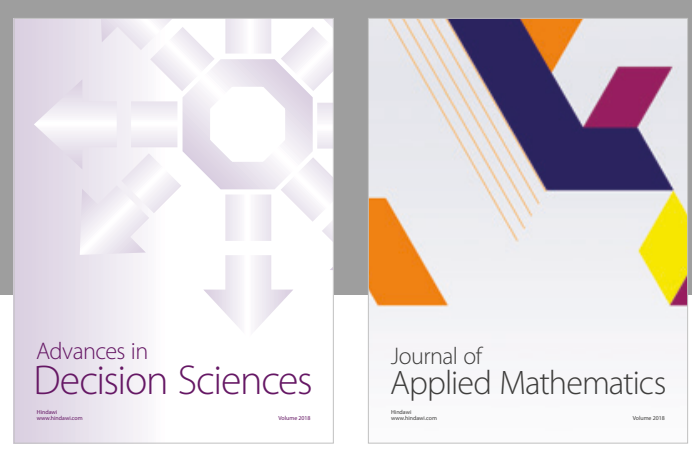

Journal of

Applied Mathematics
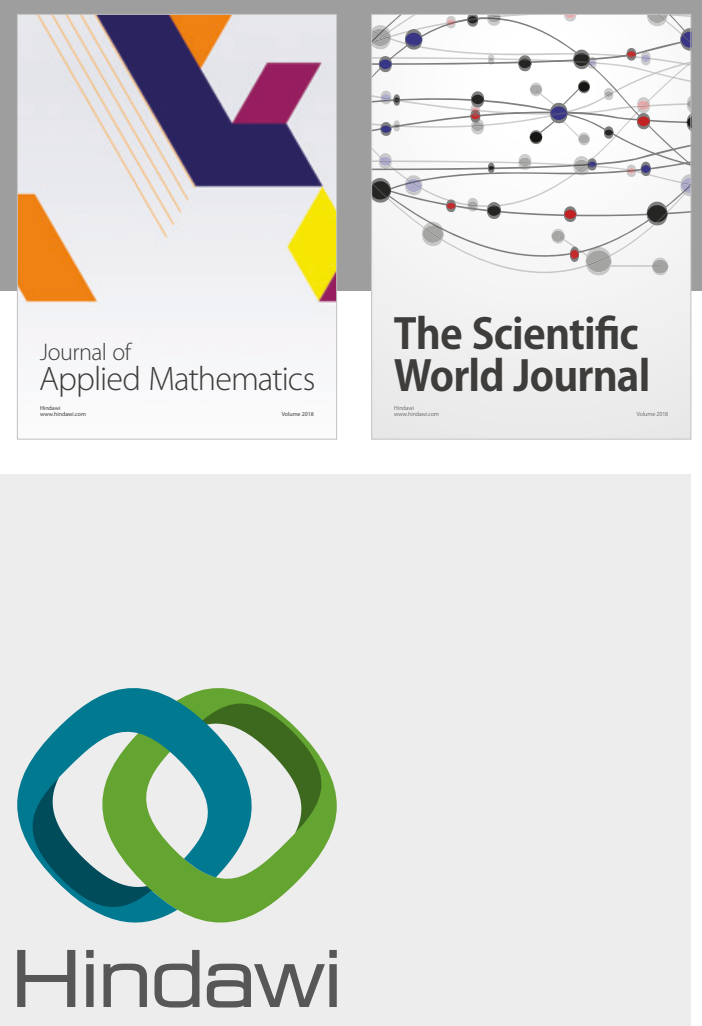

Submit your manuscripts at

www.hindawi.com

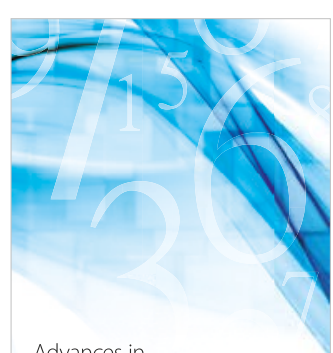

Advances in
Numerical Analysis
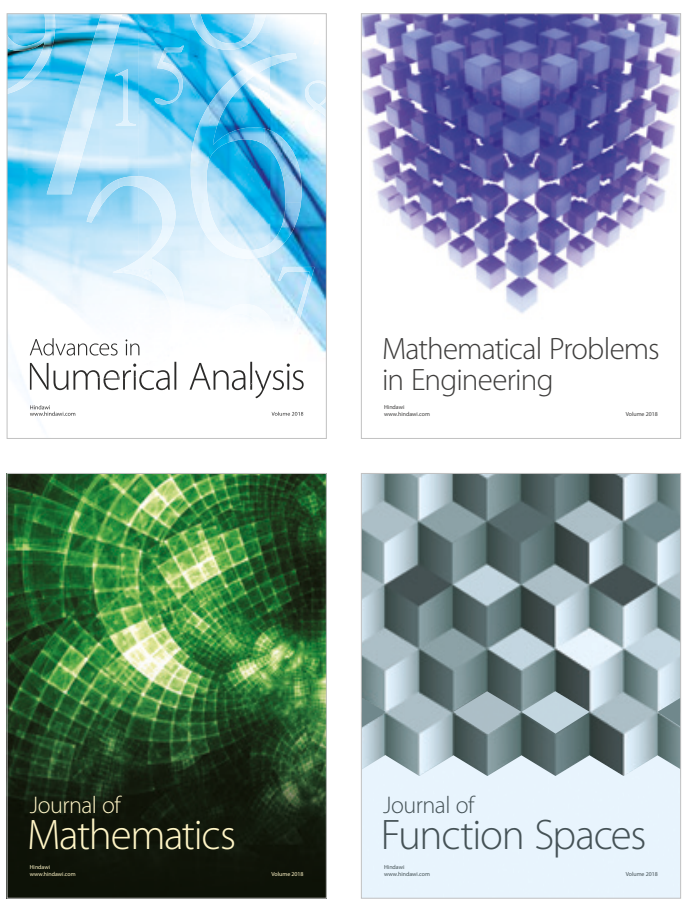

Mathematical Problems in Engineering

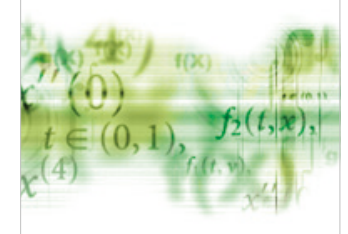

International Journal of

Differential Equations

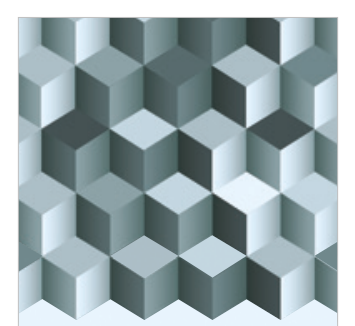

Journal of

Function Spaces

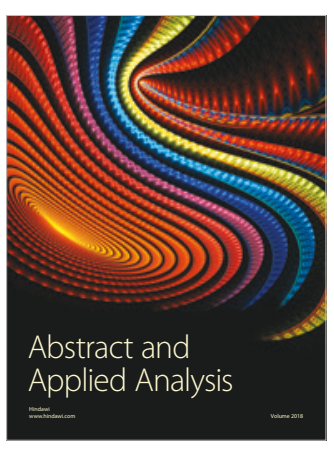

The Scientific

World Journal

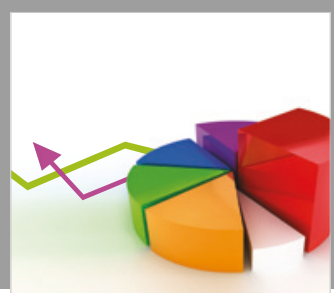

Journal of

Probability and Statistics
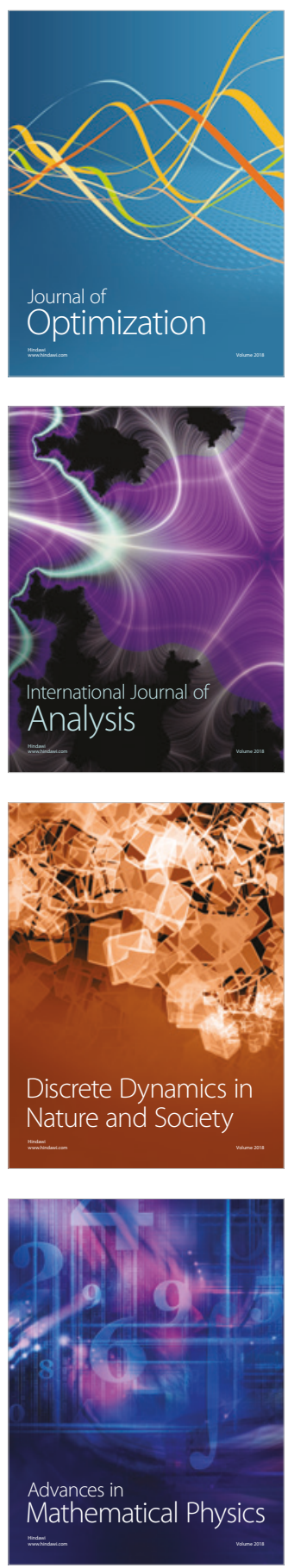